\title{
ARTICLE Cell-subtype-specific changes in adenosine pathways in schizophrenia
}

\author{
Sinead Marie O'Donovan ${ }^{1}$, Courtney Sullivan ${ }^{1}$, Rachael Koene ${ }^{1}$, Emily Devine ${ }^{1}$, Kathryn Hasselfeld ${ }^{1}$, Cassidy Lynn Moody ${ }^{1}$ and \\ Robert Erne McCullumsmith ${ }^{1}$
}

Prior work in animal models implicates abnormalities of adenosine metabolism in astrocytes as a possible pathophysiological mechanism underlying the symptoms of schizophrenia. In the present study, we sought to reverse-translate these findings back to the human brain in schizophrenia, focusing on the following questions: (1) Which components of the adenosine system are dysregulated in schizophrenia, and (2) are these changes limited to astrocytes? To address these questions, we captured enriched populations of DLPFC pyramidal neurons and astrocytes from schizophrenia and control subjects using laser capture microdissection and assessed expression of adenosine system components using qPCR. Interestingly, we found changes in enriched populations of astrocytes and neurons spanning metabolic and catabolic pathways. Ectonucleoside triphosphate diphosphohydrolase-1 (ENTPD1) and ENTPD2 mRNA levels were significantly decreased ( $p<0.05, n=16$ per group) in enriched populations of astrocytes; in pyramidal neurons equilibrative nucleoside transporter 1 (ENT1) and adenosine $A_{1}$ receptor mRNA levels were significantly decreased, with an increase in adenosine deaminase (ADA) $(p<0.05, n=16$ per group). Rodent studies suggest that some of our findings $\left(A_{1} R\right.$ and ENTPD2) may be due to treatment with antipsychotics. Our findings suggest changes in expression of genes involved in regulating metabolism of ATP in enriched populations of astrocytes, leading to lower availability of substrates needed to generate adenosine. In pyramidal neurons, changes in ENT1 and ADA mRNA may suggest increased catabolism of adenosine. These results offer new insights into the cell-subtype-specific pathophysiology of the adenosine system in this illness.

Neuropsychopharmacology (2018) 43:1667-1674; https://doi.org/10.1038/s41386-018-0028-6

\section{INTRODUCTION}

Schizophrenia is a serious, high-cost neuropsychiatric disorder that affects approximately $1 \%$ of the population worldwide [1]. The symptoms of schizophrenia can be classified as positive, negative, or cognitive [2]. Positive symptoms are characterized by delusions and hallucinations and are associated with dopamine hyperfunction [3]. Negative and cognitive symptoms are characterized by social withdrawal, anhedonia, and deterioration in memory and executive function, and are related to glutamate hypofunction $[2,4]$. The adenosine system modulates glutamate and dopamine neurotransmission via a complex network of receptors, enzymes, and transporters expressed throughout the brain on neurons and glia [5]. Adenosine metabolism is a tightly regulated process [6] and dysregulation of adenosine pathways significantly impacts the system's neuromodulatory function [7].

Boison and colleagues developed a mouse model of adenosine kinase (ADK) overexpression, resulting in a global brain adenosine deficiency and cognitive and locomotor impairments that model schizophrenia-like endophenotypes [8]. These deficits are postulated to be driven by increased expression and activity of ADK in astrocytes, leading to reduced extracellular adenosine levels $[8,9]$. In combination with models that genetically manipulate adenosine receptor expression, these studies suggest that dysregulation of adenosinergic neuromodulation of glutamatergic and dopaminergic systems contributes to the signs and symptoms found in schizophrenia [9-11]. Supporting this hypothesis, allopurinol, a xanthine oxidase inhibitor, which prevents purine degradation, has a moderate effect in improving symptoms in a subset of schizophrenia subjects when administered in addition to antipsychotic medication [12].

Postmortem studies of the adenosine system in schizophrenia, limited to only a few brain regions, have primarily focused on changes in adenosine receptor expression. Increased expression of $A_{2 A}$ receptor was reported in the hippocampus [13] and striatum $[14,15]$, while others have reported downregulation of $A_{2 A}$ receptor mRNA and protein expression in the striatum in a subset of schizophrenia subjects [16]. The protein expression levels of metabolic enzymes responsible for the generation of adenosine from ATP were also decreased in the striatum in schizophrenia [17].

There are a number of sources of extracellular adenosine. These include indirect production by enzymatic catabolism of released ATP, culminating in conversion of AMP via 5'nucleotidase (5'NT) to adenosine, a primarily astrocytic mechanism [11]. Adenosine produced by extracellular ATP released from astrocytes primarily targets $A_{1}$ receptors, which induces widespread tonic inhibition [18]. Neuronally produced adenosine preferentially activates local excitatory $A_{2 A}$ receptors [19]. Adenosine can also be generated intracellularly from AMP, following nucleotide degradation or from s-adenosyl homocysteine, and directly released by the ENT

${ }^{1}$ Department of Psychiatry, University of Cincinnati, Cincinnati, OH 45267, USA

Correspondence: Sinead Marie. O'Donovan (odonovsm@ucmail.uc.edu)

Received: 21 November 2017 Revised: 1 February 2018 Accepted: 5 February 2018

Published online: 26 February 2018 
transporters, a primarily neuronal mechanism [20, 21]. Calciumdependent adenosine release has also been identified, an effect that may be dependent on glutamate but not dopamine release $[22,23]$. In this study, we sought to examine gene expression of multiple components (enzymes, receptors, and transporters) of this diverse neuromodulatory system in schizophrenia, using a cell-level approach in postmortem DLPFC, a brain region implicated in the pathophysiology of cognitive deficits and negative symptoms found in this often-devastating illness. These components include ENTPDs, which convert ATP to ADP and AMP; 5 'nucleotidase (5'NT), an ectoenzyme that converts AMP to adenosine; ENT1, a bidirectional adenosine transporter; adenosine deaminase (ADA), which metabolizes adenosine to inosine; ADK, which phosphorylates adenosine to AMP; and the inhibitory and excitatory adenosine receptors $A_{1} R$ and $A_{2 A} R$, respectively.

\section{MATERIALS AND METHODS}

\section{Subjects}

Human brain tissue was obtained from the Maryland and Alabama Brain Collections. All cases were obtained with consent from the next of kin with IRB approved protocols. The tissue was collected from adult control $(n=16)$ and schizophrenia subjects $(n=16)$ from the dorsolateral prefrontal cortex (DLPFC). Tissue from the same subjects were used for region-level and cell-level studies. Subjects were matched for age, postmortem interval (PMI) and $\mathrm{pH}$ (Table 1). Brains were frozen at $-80^{\circ} \mathrm{C}$ until the DLPFC was dissected and stored at $-80^{\circ} \mathrm{C}$ until used. For all postmortem human cases, two independent psychiatrists established DSM-IV diagnoses based on the review of available medical records, autopsy reports, and interviews with family using the Structured Clinical Interview for DSM-IV. APD status was deemed to be "on" if they were on medication in the last 1-6 months of life.

Cell capture and quantitative polymerase chain reaction (qPCR) Laser capture microdissection (LCM) and GPCR methods are described in detail in the Supplemental Information and were conducted and validated as previously described [24-26]. Briefly, for region-level studies, RNA was extracted from $14 \mu \mathrm{m}$ DLPFC cryosections using the RNeasy Minikit (Qiagen, NL) according to the manufacturer's instructions. To conduct cell-level studies, enriched populations of pyramidal cells and astrocytes were identified and cut from superficial (layers II-III) and deep (layers $\mathrm{V}-\mathrm{VI}$ ) gray matter of the DLPFC by LCM. RNA was isolated using the PicoPure RNA isolation kit (Molecular Devices, Sunnyvale, CA, USA) according to the manufacturer's protocol. Complementary DNA (cDNA) was synthesized using a High-Capacity cDNA Reverse Transcription Kit (Applied Biosystems, Foster City, CA, USA). Taqman primers, listed in Supplementary Table 1, were used for preamplification PCR reaction and region-level and cell-level experiments using 96-well optical reaction plates (Life Technologies, USA) on an Applied Biosystems detection system (ABI SteponePlus, Applied Biosystems, Life Technologies, USA). Quality control studies confirming sample enrichment are described in Supplementary Fig. 8, as well as in prior publications [24-26].
Animal treatments

Rat haloperidol study. Rodent studies were performed in accordance with the IACUC guidelines at the University of Alabama at Birmingham. Adult male Sprague-Dawley rats (250 g) were housed in pairs and maintained on a $12 \mathrm{~h}$ light/dark cycle. Rats received $28.5 \mathrm{mg} / \mathrm{kg}$ haloperidol-decanoate [27, 28] or vehicle (sesame oil) by intramuscular injection every 3 weeks for 9 months

Rat PMI study. Rodent studies were performed in accordance with the IACUC guidelines at the University of Cincinnati. LCMqPCR experiments in antipsychotic treated rats for selected dependent measures were performed similar to human studies [25]. mRNA levels for selected dependent measures were also assayed in the frontal cortex of adult male Sprague-Dawley rats $(250 \mathrm{~g})$ that simulate varying PMls of $0,12,24$, and $48 \mathrm{~h}(n=3$ per time point).

\section{qPCR in rodent brain}

Gene expression targets that were significantly altered in postmortem DLPFC were compared in haloperidol-treated $(n=$ 10) and control $(n=10)$ rats. The frontal cortex of each rodent was cryo-sectioned ( $14 \mu \mathrm{m}$ sections) following removal of the olfactory bulbs and frontal pole. For cell-level experiments, enriched populations containing 500 pyramidal cells or astrocytes were cut from Nissl stained sections by LCM. ENT1 expression was significantly correlated with PMI in the human study. As an additional control for this variable, ENT1 expression was examined in the rat PMI time course study at region-level. RNA was isolated using the Arcturus PicoPure RNA isolation kit (Applied Biosystems, Foster City, CA, USA) according to the manufacturer's protocol. cDNA was then reverse transcribed and analyzed by QPCR as described. The primers used are listed in Supplementary Table 2.

\section{Inosine assay}

Inosine levels were measured at the region-level in control $(n=$ $16)$ and schizophrenia $(n=16)$ DLPFC. Tissue was prepared and the assay was carried out according to the manufacturer's instructions (MAK100, Sigma-Aldrich, USA) with minor adjustments. Briefly, standards and samples were run in a final volume of $40 \mu \mathrm{l}$ with equal volume of sample buffer. The amount of sample loaded per well was equivalent to $6 \mu \mathrm{g}$ of protein. Samples and standards were run in duplicate. The assay was read using a fluorescent plate reader (Envision 2103, PerkinElmer, USA) at 535 $\mathrm{nm}$ excitation $/ 590 \mathrm{~nm}$ emission.

Data analysis

Alpha $=0.05$ for all statistical tests. Data were analyzed with Statistica (Statsoft, Tulsa, OK, USA) and Graphpad Prism 6, (GraphPad Software, La Jolla California USA, www.graphpad. com). All data sets were tested for normal distribution (D'Agostino and Pearson omnibus normality test) and homogeneity of variance ( $F$-test). Outliers $>2$ standard deviations from the mean were excluded.

Table 1. Demographics of Maryland Brain Collection subjects used in study

\begin{tabular}{llllllll}
\hline & $N$ & Sex & PMI & Age & pH & RIN & Medication \\
\hline Control & 16 & $2 \mathrm{~F} / 14 \mathrm{M}$ & $12.9 \pm 4.0(7-20)$ & $43 \pm 10(23-60)$ & $6.7 \pm 0.2(6.1-6.97)$ & $5.6 \pm 1.39(3.8-7.6)$ & N/A \\
Schizophrenia & 16 & $2 \mathrm{~F} / 14 \mathrm{M}$ & $14.8 \pm 5.5(6-25)$ & $45 \pm 12(20-67)$ & $6.7 \pm 0.33(6.07-7.12)$ & $6.2 \pm 1.28(3.5-7.7)$ & On/off/unknown (13/1/2) \\
\hline
\end{tabular}

Medication, on/off/unknown [number of subjects on: 11 typical antipsychotics/ 2 atypical antipsychotics]. Data range in parentheses. Data presented as mean $+/$ - standard deviation

$F$ female, $M$ male, $N$ number of subjects, $P M I$ postmortem interval (hours), RIN RNA integrity number 

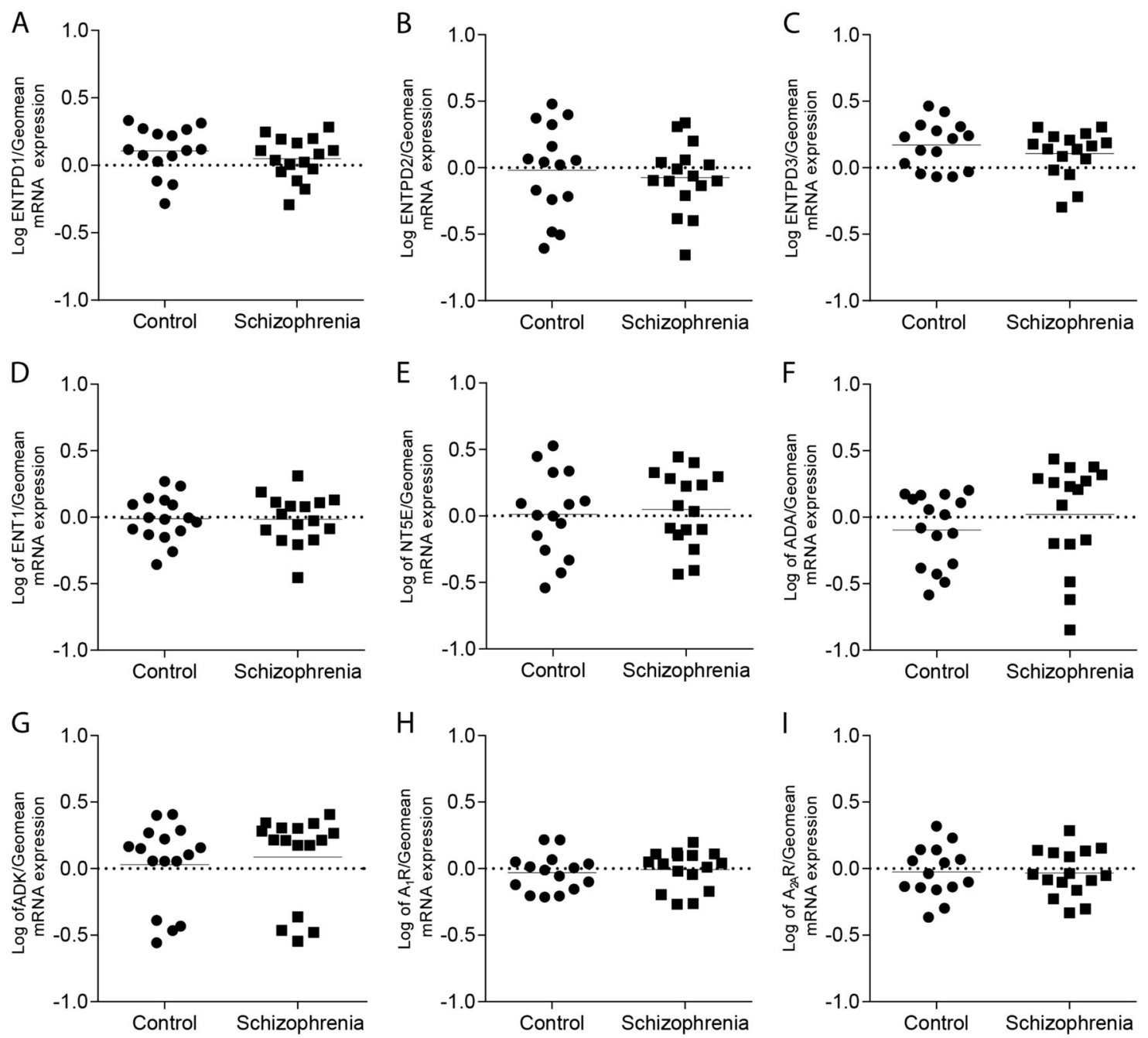

Fig. 1 Region-level expression of adenosine metabolism pathway components. There was no change in gene expression of a ENTPD1, $\mathbf{b}$ ENTPD2, c ENTPD3, d ENT1, e NT5E, f ADA, g ADK, $\mathbf{h} A_{1} R$, and $\mathbf{i} A_{2 A} R$ in schizophrenia subjects compared to controls following Student's $t$-test. Data mean + / - SEM, $n=14-16 /$ group. ENTPD ectonucleoside triphosphate diphosphohydrolase, ENT equilibrative nucleoside transporter, NT5E 5'nucleotidase (5'NT), ADA adenosine deaminase, ADK adenosine kinase, $A_{1 / 2 A} R$ adenosine ${ }_{1 / 2 A}$ receptor

Human studies. Data were log transformed. Correlation analyses were performed to determine associations between transcript expression and age, PMI, and RIN value. Analysis of covariance (ANCOVA) was performed if significant correlations were found. If no correlations were present, data were analyzed with Student's $t$ test or Mann-Whitney test for non-parametric analysis. Secondary analyses were performed to assess the effects of sex and medication on the dependent measures. Correlation analyses were conducted to quantify the association of target mRNA expression and PMI.

Rodent studies. Data was analyzed by Student's t-test or with other tests if the assumption of homogeneity of variance (Welch's t-test) or normal distribution (Mann-Whitney test) were not met.

\section{RESULTS}

The mRNA expression levels of enzymes involved in the extracellular generation of adenosine, ectonucleoside triphosphate diphosphohydrolase (ENTPD)1-3, ADA, ADK, adenosine $A_{1}$ receptor $\left(A_{1} R\right)$, adenosine $A_{2 A}$ receptor $\left(A_{2 A} R\right)$, 5'nucleotidase $\left(5^{\prime} \mathrm{NT}\right)$ and the adenosine transporter equilibrative nucleoside transporter (ENT1) were measured in tissue homogenates (regionlevel studies) and in enriched populations of astrocytes and pyramidal neurons (cell-level studies) in the DLPFC in schizophrenia.

Region-level studies

In the DLPFC tissue homogenates there was a significant association between gene expression of ENTPD1 and ADK with RIN, but not age or PMI. There were no significant associations between expression of the target mRNA ENTPD2, ENTPD3, 5'NT, ENT1, ADA, $A_{1} R$ and $A_{2 A} R$ and age, $p H, P M I$, or RIN values.

There were no significant changes in mRNA expression for ENTPD1 (ANCOVA: $F_{(1,28)}=0.221, p=0.642$ ) or ADK (ANCOVA: $\left.F_{(1,29)}=0.025, p=0.876\right)$. There were no significant changes in mRNA expression for ENTPD2 $(p=0.622)$, ENTPD3 $(p=0.244)$, ENT1 $(p=0.966), 5$ 'NT $(p=0.734)$, ADA $(p=0.333), \mathrm{A}_{1} \mathrm{R}(p=$ $0.0629)$ or $\mathrm{A}_{2 \mathrm{~A}} \mathrm{R}$ ( $p=0.913$ ) (Fig. 1).

Astrocyte population

In a population of enriched astrocytes from DLPFC, there was a significant association between expression of ENT1 and ENTPD3 mRNA expression and RIN, but not age or PMI. There were no significant associations between gene expression levels of ENTPD1, ENTPD2, $A_{1} R, A_{2 A} R, 5 ' N T, A D A$ and $A D K$ and age, $p H$, $\mathrm{PMI}$, or RIN values. 

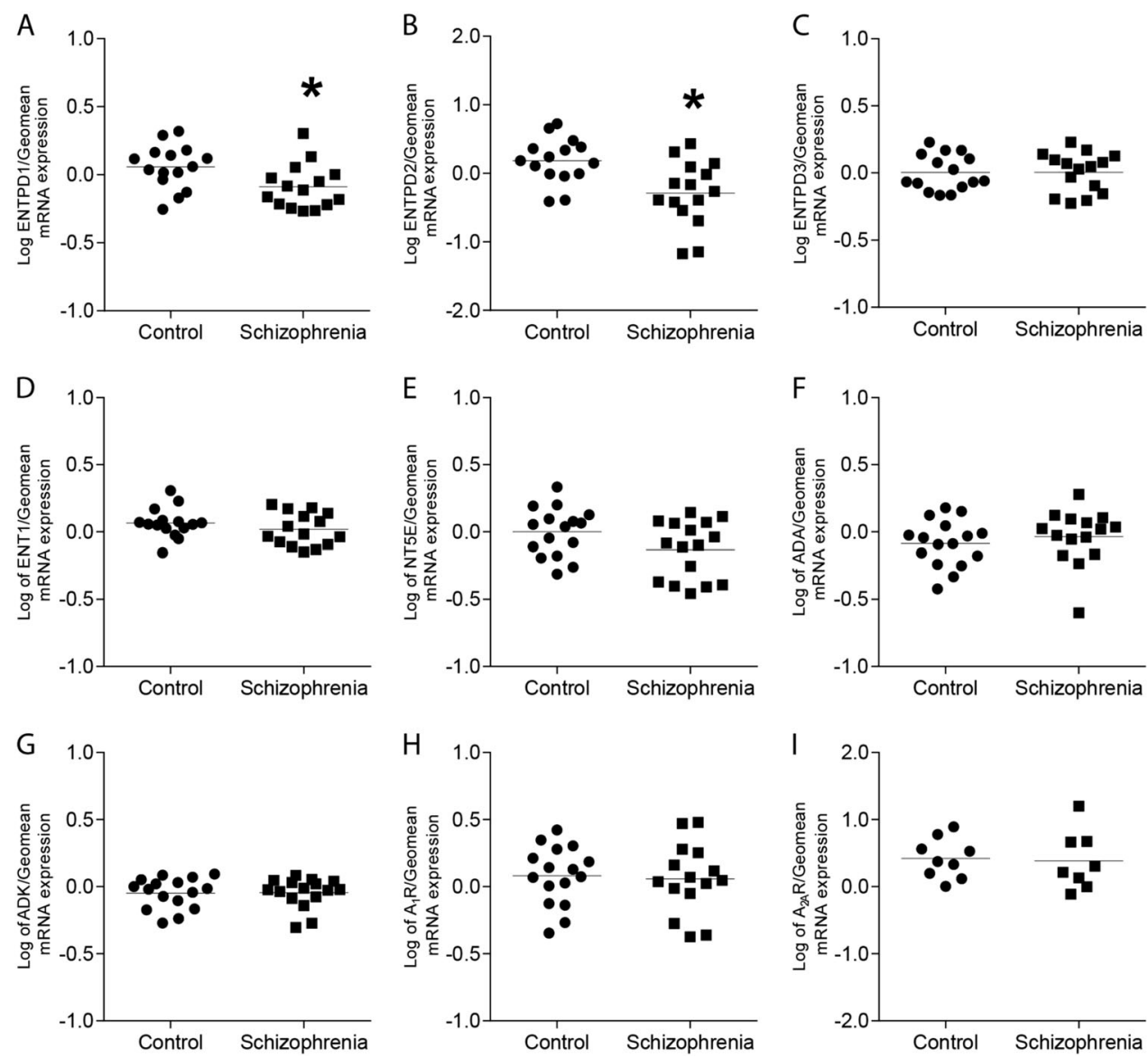

Fig. 2 Enriched astrocyte population expression of adenosine metabolism pathway components. There was a significant decrease in gene expression of a ENTPD1 $(p<0.05)$ and $\mathbf{b}$ ENTPD2 $(p<0.05)$. Following Student's $t$-test, there was no significant change in gene expression of $\mathbf{c}$ ENTPD3, d ENT1, e NT5E, f ADA, g ADK, $\mathbf{h} A_{1} R$, and $\mathbf{i} A_{2 A} R$ in schizophrenia subjects compared to controls. Data mean $+/-S E M, n=8-16 /$ group. ENTPD ectonucleoside triphosphate diphosphohydrolase, ENT equilibrative nucleoside transporter, NT5E 5'nucleotidase (5'NT), ADA adenosine deaminase, $A D K$ adenosine kinase, $A_{1 / 2 A} \mathrm{R}$ adenosine ${ }_{1 / 2 A}$ receptor

There was no significant difference between schizophrenia and control expression of ENT1 mRNA (ANCOVA: $F_{(1,27)}=0.271, p=$ $0.607)$ or ENTPD3 $\left(F_{(1,27)}=0.829, p=0.371\right)$ (Fig. 2d, c). There was a significant decrease in ENTPD1 $\left(t_{(28)}=2.5, p=0.0189\right)$ and ENTPD2 $\left(t_{(28)}=3.2, p=0.0034\right)$ mRNA expression (Fig. $2 a$, b). There was no significant effect of sex or medication on expression in schizophrenia compared to control. No statistically significant changes in mRNA expression were detected for $5^{\prime} \mathrm{NT}(p=0.065)$, $\mathrm{A}_{1} \mathrm{R}(p=0.773)$, and $\mathrm{A}_{2 \mathrm{~A}} \mathrm{R}(p=0.842)$ (Fig. $\left.2 \mathrm{e}, \mathrm{h}, \mathrm{i}\right)$ or ADA $(p=$ $0.332)$ and ADK $(p=0.924)$, which were analyzed by Mann-Whitney test as data distribution was not normal following log transformation (Fig. 2f, g).

Pyramidal cell population

In a population of enriched pyramidal neurons from DLPFC, there was a significant association between the expression of ENT1 and PMI, but not age, pH, or RIN and between ENTPD2 and age, but not RIN, $\mathrm{pH}$, or PMI. There were no significant associations between gene expression levels of ENTPD1, ENTPD3, 5'NT, ADA, ADK, $A_{1} R, A_{2 A} R$ and age, $\mathrm{pH}, \mathrm{PMI}$, or RIN values.

There was a significant decrease in ENT1 mRNA (ANCOVA: $F_{(1,27)}$ $=11.326, p=0.002$ ) but no significant difference in ENTPD2 expression (ANCOVA: $F_{(1,22)}=3.544, p=0.0731$ ). There was a significant decrease in $A_{1} R\left(t_{(29)}=2.3, p=0.032\right)$ and a significant increase in ADA $\left(t_{(30)}=2.4, p=0.021\right)$ mRNA expression in schizophrenia compared to controls (Fig. 3d, h, f). There was no significant effect of sex or medication on expression of ENT1, $A_{1} R$, or ADA. No statistically significant changes in mRNA expression were detected for ENTPD1 $(p=0.904), \operatorname{ENTPD3}(p=0.071), 5$ 'NT $(p=0.829), \mathrm{A}_{2 \mathrm{~A}} \mathrm{R}(p=0.464)$, or ADK $(p=0.276)$ (Fig. 3a-c, e, g, i).

PMI and RIN correlation analyses

There were no significant associations between gene transcripts and PMI in the enriched astrocyte population (Supplementary Fig. 1). In an enriched population of pyramidal neurons, there was a significant negative association between ENT1 mRNA expression and PMI ( $r=-0.4542, p=0.012$; Supplementary Fig. 2D) but not between other gene transcripts and PMI (Supplementary Fig. 2).

In an enriched population of astrocytes, there was a significant negative association between ENTPD3 and RIN $(r=-0.51, p=$ $0.004)$ and ENT1 mRNA expression and RIN $(r=-0.43, p=0.0187)$ but not between other gene transcripts and RIN (Supplementary Fig. 3). There were no significant associations between gene transcripts and RIN in the in the pyramidal neuron population (Supplementary Fig. 4).

Inosine assay

No changes in inosine levels were detected in DLPFC tissue homogenates in schizophrenia vs. control subjects (Supplementary Fig. 5).

\section{Rodent studies}

To assess the effects of chronic antipsychotic treatment on our dependent measures, gene expression of significantly altered mRNA targets from our gene studies were measured in rats treated for 9 months with haloperidol-decanoate. In an enriched 

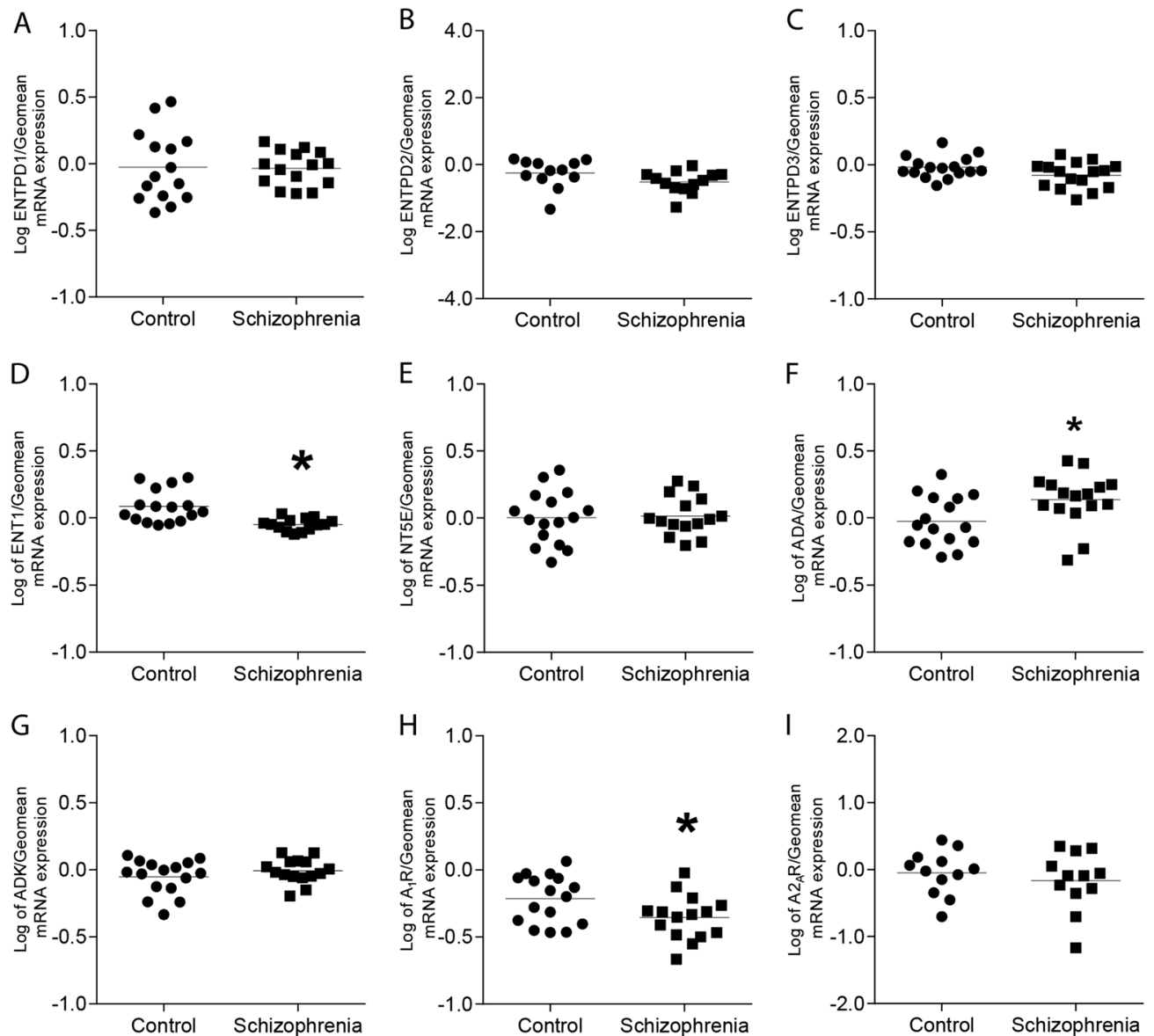

Fig. 3 Enriched pyramidal cell population expression of adenosine metabolism pathway components. Following one-way ANCOVA, there was a significant decrease in gene expression of $\mathbf{d}$ ENT1 $(p<0.05)$. Student's $t$-test analysis revealed a significant increase in expression of $\mathbf{f}$ ADA $(p$ $<0.05)$ and $\mathbf{h ~} A_{1} R(p<0.05)$. There was no significant change in gene expression of a ENTPD1, $\mathbf{b}$ ENTPD2, $\mathbf{c}$ ENTPD3, e NT5E, g ADK, and i $A_{2 A} R$ in schizophrenia subjects compared to controls. Data mean $+/-$ SEM, $n=14$ 16/group. ENTPD ectonucleoside triphosphate diphosphohydrolase, ENT equilibrative nucleoside transporter, NT5E 5'nucleotidase (5'NT), ADA adenosine deaminase, ADK adenosine kinase, $A_{1 / 2 A} R$ adenosine ${ }_{1 / 2 \mathrm{~A}}$ receptor

population of astrocytes there was no significant effect of medication on ENTPD1 ( $p>0.05$, Fig. 4a) or 5'NT ( $p>0.05$, Fig. $4 c$ ). There was a significant decrease in ENTPD2 mRNA expression (Welch-corrected $t_{(13)}=2.3, p=0.042$, Fig. 4b).

There was no significant effect of medication on ENT1 expression or ADA expression in an enriched population of pyramidal neurons (Fig. 4d, e). There was a decrease in $A_{1} R$ expression (Welch-corrected $t_{(8.8)}=3.8, p=0.004$ ) in an enriched population of pyramidal neurons (Fig. $4 \mathrm{f}$ ).

ENT1 expression and PMI were significantly correlated in pyramidal neurons (Supplementary Fig. 2D). Therefore, the effect of PMI on ENT1 expression was examined in a rat model of PMI. There was no significant effect of PMI on ENT1 gene expression at $12 \mathrm{~h}, 24 \mathrm{~h}$ or $48 \mathrm{~h}$ compared to $0 \mathrm{~h}$ in the rat frontal cortex (Supplementary Fig. 6).

\section{DISCUSSION}

Boison and colleagues speculate that hypofunction of the neuromodulator adenosine results in dysregulation of the glutamate and dopamine neurotransmission systems, which are central to the pathophysiology of schizophrenia [9]. Evidence supporting this hypothesis comes from a number of animal model studies [9], but there is a paucity of information on the pathways regulating extracellular adenosine generation in human disease. Thus, in this study, we identified components of the adenosine system (enzymes, receptors, and transporters) involved in the extracellular generation of adenosine that have dysregulated gene expression in postmortem DLPFC in schizophrenia. Our results also extend the current understanding of the hypothesis from being a primarily glial defect, to include perturbations in the pathway that generates extracellular adenosine in neurons. Overall, this study suggests that there is extensive, cell-subtype-specific dysfunction in gene expression of the metabolic cascade that generates extracellular adenosine in schizophrenia.

Extracellular adenosine is mainly generated in two ways. ATP is released into the extracellular space and converted to adenosine via a series of enzymatic steps. Alternatively, adenosine is directly released from cells via equilibrative nucleoside transporters (ENTs) $[7,11]$. Indirect generation of adenosine via conversion of ATP is more typical of glial cells, while direct adenosine release is more typical of neurons, although the enzymes required to generate adenosine and the ENT transporters are found in both cell types $[7,29]$. The relative contributions of directly released and indirectly generated adenosine and whether its source is neuronal or astrocytic are not fully understood. However, in pathological conditions like schizophrenia, the results of this study suggest that diverse cell-subtype-specific mechanisms may contribute to dysregulation of the adenosine metabolism cascade (Fig. 5).

In astrocytes, ENTPD1 and ENTPD2 generate adenosine by sequentially hydrolyzing ATP to ADP and AMP in the extracellular space [30]. ENTPD-mediated catabolism of ATP terminates its 


\section{Astrocytes}

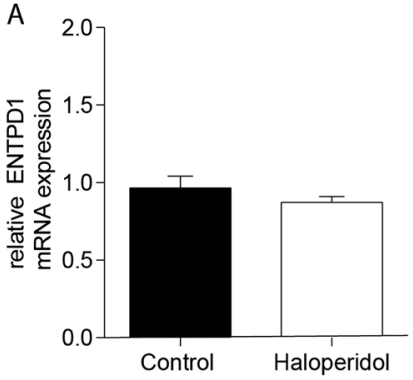

Pyramidal Neurons

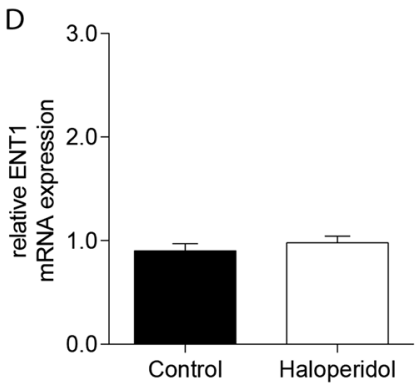

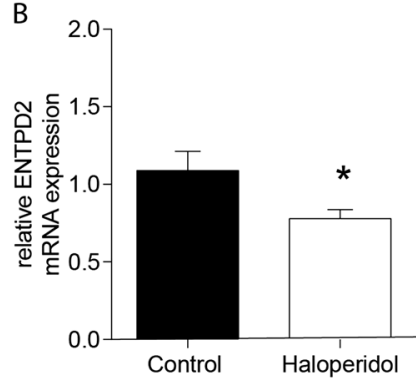

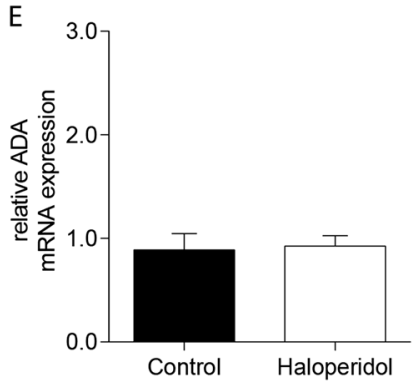

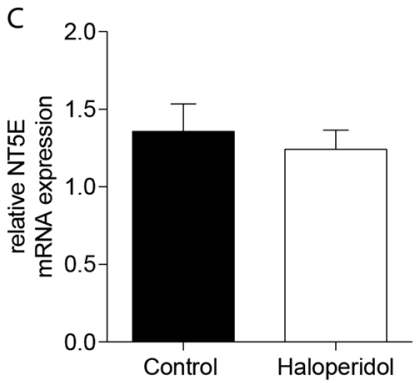

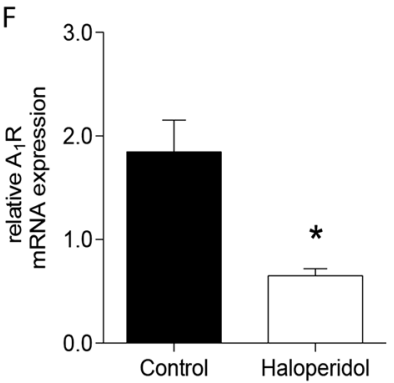

Fig. 4 Relative mRNA expression of significantly altered adenosine system genes in haloperidol-treated rats. In an enriched population of astrocytes, there was no significant effect of antipsychotic medication on a ENTPD1 or c NT5E mRNA levels following Student's $t$-test. There was a significant decrease in b ENTPD2 levels $(p<0.05) . n=9-10 /$ group. In an enriched population of pyramidal neurons, there was no significant effect of antipsychotic medication on d ENT1 or e ADA mRNA levels following Student's $t$-test. f $A_{1} R$ levels were significantly reduced following chronic haloperidol administration. $n=5-10 /$ group. Data presented as mean $+/-S E M$. ADA adenosine deaminase; $A_{1} R$ adenosine 1 receptor; ENT1 ectonucleoside transporter 1; ENTPD ectonucleoside triphosphate diphosphohydrolase; NT5E 5'nucleotidase $\left(5^{\prime} \mathrm{NT}\right)$

action as a signaling molecule but initiates ADP and adenosine signaling responses [31]. In cardiac fibroblasts, endogenous ENTPD activity maintains tissue homeostasis by modulating stimulatory ATP release and inhibitory ATP hydrolysis [32]. This provides a balancing mechanism that maintains extracellular adenosine and ATP levels. The authors postulate that blockade of ENTPD activity reduced adenosine signaling and prolonged ATP signaling. It follows that attenuated ENTPD expression may significantly impact adenosine system signaling and neuromodulatory activities. In this study, ENTPD1 and ENTPD2 gene expression levels were significantly reduced in an enriched population of astrocytes in schizophrenia (Fig. 2a, b). In the striatum, ENTPD enzyme activity levels were downregulated in schizophrenia [17]. Reduced levels of ENTPD could have a significant impact on the amount of substrate available for conversion to adenosine, affecting adenosine and purinergic receptor activation.

ENT1 is a non-concentrative bidirectional nucleoside transporter that equilibrates adenosine concentration across membranes [33]. ENT1 facilitates both influx and efflux of adenosine. Thus, adenosine transporter blockers can increase extracellular adenosine levels, leading to activation of $A_{1}$ receptors and increased $A_{1}$ receptor-mediated inhibition, or reduce extracellular adenosine [20], as with ENT1 knockout mice, which have reduced adenosinemediated inhibition of EPSCs [34]. In schizophrenia, ENT1 mRNA levels were significantly reduced in pyramidal neurons (Fig. $3 \mathrm{~d}$ ). Consistent with this finding, a decrease in ENT1 protein levels was found in the superior temporal gyrus in elderly schizophrenia subjects [35]. No change in ENT1 protein was found in the anterior cingulate cortex in the same study. ENT1 allows for fast, direct release of adenosine under normal conditions and is responsible for approximately $40 \%$ of extracellular adenosine release [36]. Lovatt et al. [23] showed ENT-dependent release of adenosine in response to active spiking neurons, resulting in $A_{1}$ receptor activation and suppression of excitatory transmission. This effect was independent of $5^{\prime} \mathrm{NT}$ activity, indicating it was not due to extracellularly metabolized ATP, but directly released adenosine from neurons [23]. Thus, reduced neuronal ENT1 expression could result in decreased levels of neuronally released adenosine and lower extracellular adenosine levels, contributing to dysregulation of the adenosine system in schizophrenia.

ADA provides a means of metabolically inactivating adenosine. ADA irreversibly deaminates adenosine to inosine, which is unavailable for signaling [37]. We found an increase in ADA levels in an enriched population of pyramidal neurons (Fig. 3f), suggesting increased catabolism of adenosine that may contribute to dysregulation of the adenosine system in schizophrenia. ADA activity levels in serum are significantly increased in schizophrenia subjects, although it is unclear whether this is solely a disease effect or the influence of antipsychotic medication [38]. Schizophrenia subjects are less likely to have a low activity variant of $A D A, A D A 1 * 2, a G$ to $A$ polymorphism of $A D A$ that results in $20-30 \%$ lower enzyme activity levels [39]. This results in lower levels of adenosine in schizophrenia subjects due to the higher activity variant of ADA. To investigate a possible consequence of increased ADA expression, inosine levels were assayed in this study. No changes in the concentration of inosine at the region-level in tissue homogenates were detected. However, region-level measurements of metabolites may not be sensitive enough to detect cell-level or compartment-specific changes in inosine expression [40].

$A_{1} R$ gene expression was significantly downregulated in an enriched pyramidal neuron population in schizophrenia. $A_{1} R s$ mediate inhibition of synaptic transmission following activation by adenosine under basal conditions in excitatory synapses $[33,41]$. Limited animal studies of $A_{1} R$ genetic deletion resulted in animals 
In Schizophrenia

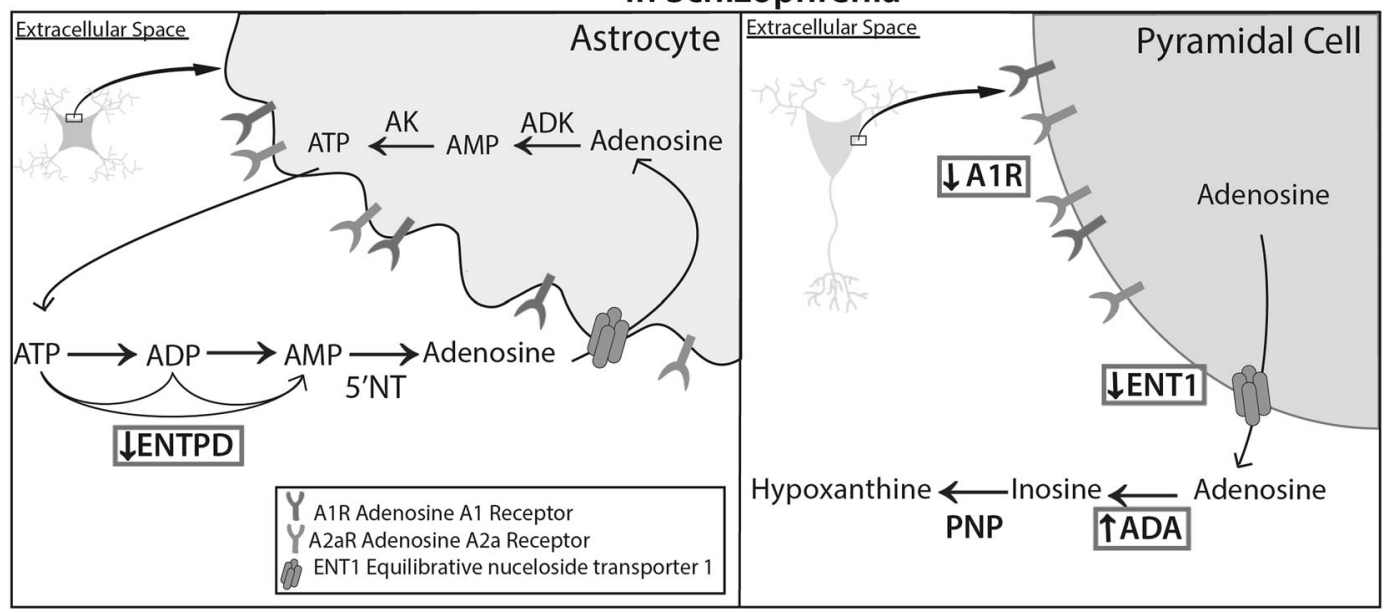

Fig. 5 Summary of altered gene expression of adenosine metabolism pathway in astrocytes and pyramidal neurons in schizophrenia. In an enriched population of astrocytes, ENTPD1 and ENTPD2, enzymes responsible for the dephosphorylation of ATP and ADP to AMP, the substrate for adenosine, are significantly reduced. The indirect generation of adenosine, from ATP release by astrocytes, is likely reduced in schizophrenia. In an enriched population of neurons, ADA, an enzyme which irreversibly converts adenosine to inosine, is significantly increased. ENT1, a bidirectional nucleoside transporter, and the adenosine $A_{1}$ receptor are also significantly reduced. The direct release and availability of adenosine generated in neurons is likely reduced in schizophrenia. Adenosine hypofunction is strongly implicated in schizophrenia, with cell-specific deficits in the mechanisms of adensoine generation in the DLPFC. ADA adenosine deaminase, ADK adenosine kinase, AK adenylate kinase, DLPFC dorsolateral prefrontal cortex, ENTPD ectonucleoside triphosphate diphosphohydrolase, ENT equilibrative nucleoside transporter, PNP purine nucleoside phosphorylase, 5'NT 5'nucleotidase (5'NT)

that displayed greater aggression and anxiety behavior but had no effect on spatial reference and working memory [42]. Previous postmortem work has identified no change in $A_{1} R$ gene or protein expression in the striatum in schizophrenia [16], although this study was conducted at the region-level, which may have masked cell-level changes in $A_{1} R$ expression [43].

The cell-specific distribution of the adenosine cascade gene targets corroborates reports from RNAseq analysis of different cell types in mouse cortex [44]. For example, ENTPD2 is highly expressed in astrocytes, thus changes in expression levels may have a significant impact on adenosine metabolism in this cell type. ENTPD1 is highly expressed in microglia, with lower expression in astrocytes under normal conditions; therefore, changes in expression levels in astrocytes may have only moderate effects in these cells.

Antipsychotic medication may account for some of the gene expression changes described in this study. To address this concern, the effects of chronic (9-month) administration of the typical antipsychotic haloperidol-decanoate on rat frontal cortex were studied. This is representative of the schizophrenia subject population, with $11 / 16$ subjects administered typical antipsychotics from a total 14/16 subjects known to be administered antipsychotic medication (Table 1). ENTPD1 (enriched populations of astrocytes), ADA, and ENT1 (enriched populations of pyramidal neurons) levels were not altered in rats treated chronically with haloperidol-decanoate. However, ENTPD2 and $A_{1} R$ levels were significantly decreased. Haloperidol also reduced ATP hydrolysis and ENTPD2 gene expression in zebrafish brain $[45,46]$. A decrease in $A_{1} R$ expression may indicate reduced inhibition of glutamate release, leading to increased excitatory signaling in response to hypoglutamatergic function associated with schizophrenia [9]. These results represent the effects of chronic dopamine $D_{2}$ receptor antagonism on the adenosine metabolism cascade, and suggest a novel mechanism of action for these drugs in treating schizophrenia.

A possible limitation of this study is the sample size. LCM-qPCR is a time-consuming method and the experimental design is balanced to collect the number of samples required for sufficiently powered analyses, in a timely manner. As most schizophrenia subjects were on antipsychotic medications like haloperidol, it is not possible to meaningfully compare the effects with subjects off antipsychotics in this dataset. An additional limitation is that gene expression changes are measured in populations of cells that are highly enriched [2426], but may contain neuropil, processes, or other small cells including interneurons. ENT1 expression is sensitive to hypoxic conditions. Therefore, ENT1 was statistically analyzed with PMI as a covariate to control for this common feature of postmortem tissue. A rodent study to control for the effects of PMI on gene expression found no effect. Finally, while we can speculate on the effects of cell-specific changes in gene expression of adenosine metabolism targets, future studies using experimental models are needed to test mechanistic hypotheses to explain these postmortem observations and determine the functional consequences of dysregulation of adenosine metabolism pathways in schizophrenia.

In summary, this study provides evidence for dysregulated gene expression of the adenosine metabolic cascade in schizophrenia. Increased ADK activity in astrocytes is hypothesized to reduce extracellular adenosine, with subsequent hypofunction of the adenosine neurotransmitter system [5, 9]. There was no change in ADK mRNA expression in this study. However, in our study, reduced expression of the astrocytic ENTPD cascade is a more likely cause of perturbations in the adenosine system in schizophrenia. Altered expression and activity of ENTPD in schizophrenia was previously reported, albeit in the striatum [17]. In addition, our results suggest a role for neuron-driven reduction of extracellular adenosine. Changes in adenosine pathway targets in pyramidal neurons, including reduced transport of adenosine to the extracellular space via ENT1, and increased catabolism of adenosine to inosine by the enzyme ADA, may contribute to dysregulation of the adenosine metabolism system in schizophrenia. Finally, expression changes in adenosine pathway gene targets in a haloperidol medication-control study suggest that antipsychotic drugs modulate some components of this pathway, offering additional insight into the role of adenosine system dysfunction in schizophrenia. 


\section{ACKNOWLEDGEMENTS}

We thank Dr. Terry Kirley for his assistance and useful discussions on ENTPDs.

\section{ADDITIONAL INFORMATION}

Supplementary information accompanies this paper at https://doi.org/10.1038/ s41386-018-0028-6.

Competing interests: The authors declare no competing interests.

Publisher's note: Springer Nature remains neutral with regard to jurisdictional claims in published maps and institutional affiliations.

\section{REFERENCES}

1. Freedman R. Schizophrenia. N Engl J Med. 2003;349:1738-49.

2. van Os J, Kapur S. Schizophrenia. Lancet. 2009;374:635-45.

3. Mackay AV, Iversen LL, Rossor M, Spokes E, Bird E, Arregui A, et al. Increased brain dopamine and dopamine receptors in schizophrenia. Arch Gen Psychiatry. 1982;39:991-7.

4. Olney JW, Newcomer JW, Farber NB. NMDA receptor hypofunction model of schizophrenia. J Psychiatr Res. 1999;33:523-33.

5. Lara DR, Dall'Igna OP, Ghisolfi ES, Brunstein MG. Involvement of adenosine in the neurobiology of schizophrenia and its therapeutic implications. Prog Neuropsychopharmacol Biol Psychiatry. 2006:30:617-29.

6. Ipata PL, Camici M, Micheli V, Tozz MG. Metabolic network of nucleosides in the brain. Curr Top Med Chem. 2011;11:909-22.

7. Cunha RA. How does adenosine control neuronal dysfunction and neurodegeneration? J Neurochem. 2016;139:1019-55.

8. Yee BK, Singer P, Chen JF, Feldon J, Boison D. Transgenic overexpression of adenosine kinase in brain leads to multiple learning impairments and altered sensitivity to psychomimetic drugs. Eur J Neurosci. 2007;26:3237-52.

9. Boison D, Singer P, Shen HY, Feldon J, Yee BK. Adenosine hypothesis of schizophrenia-opportunities for pharmacotherapy. Neuropharmacology. 2012;62:1527-43.

10. Boison D. Adenosine kinase: exploitation for therapeutic gain. Pharmacol Rev. 2013;65:906-43.

11. Boison D, Chen JF, Fredholm BB. Adenosine signaling and function in glial cells. Cell Death Differ. 2010;17:1071-82

12. Brunstein MG, Ghisolfi ES, Ramos FL, Lara DR. A clinical trial of adjuvant allopurinol therapy for moderately refractory schizophrenia. J Clin Psychiatry. 2005;66:213-9.

13. Hwang Y, Kim J, Shin JY, Kim Jl, Seo JS, Webster MJ, et al. Gene expression profiling by mRNA sequencing reveals increased expression of immune/inflammation-related genes in the hippocampus of individuals with schizophrenia. Transl Psychiatry. 2013;3:e321.

14. Deckert J, Brenner M, Durany N, Zochling R, Paulus W, Ransmayr G, et al. Upregulation of striatal adenosine $\mathrm{A}(2 \mathrm{~A})$ receptors in schizophrenia. Neuroreport. 2003;14:313-6.

15. Kurumaji A, Toru M. An increase in [3H] CGS21680 binding in the striatum of postmortem brains of chronic schizophrenics. Brain Res. 1998;808:320-3.

16. Villar-Menendez I, Diaz-Sanchez S, Blanch M, Albasanz JL, Pereira-Veiga T, Monje $A$, et al. Reduced striatal adenosine A2A receptor levels define a molecular subgroup in schizophrenia. J Psychiatr Res. 2014;51:49-59.

17. Aliagas E, Villar-Menendez I, Sevigny J, Roca M, Romeu M, Ferrer I, et al. Reduced striatal ecto-nucleotidase activity in schizophrenia patients supports the "adenosine hypothesis". Purinergic Signal. 2013;9:599-608.

18. Pascual O, Casper KB, Kubera C, Zhang J, Revilla-Sanchez R, Sul JY, et al. Astrocytic purinergic signaling coordinates synaptic networks. Science. 2005;310:113-6.

19. Cunha RA. Different cellular sources and different roles of adenosine: A1 receptor-mediated inhibition through astrocytic-driven volume transmission and synapse-restricted A2A receptor-mediated facilitation of plasticity. Neurochem Int. 2008;52:65-72

20. Latini S, Pedata F. Adenosine in the central nervous system: release mechanisms and extracellular concentrations. J Neurochem. 2001;79:463-84.

21. Brundege JM, Dunwiddie TV. Metabolic regulation of endogenous adenosine release from single neurons. Neuroreport. 1998;9:3007-11.

22. Klyuch BP, Dale N, Wall MJ. Deletion of ecto-5'-nucleotidase (CD73) reveals direct action potential-dependent adenosine release. J Neurosci: Off J Soc Neurosci. 2012:32:3842-7.
23. Lovatt D, Xu Q, Liu W, Takano T, Smith NA, Schnermann J, et al. Neuronal adenosine release, and not astrocytic ATP release, mediates feedback inhibition of excitatory activity. Proc Natl Acad Sci USA. 2012;109:6265-70.

24. McCullumsmith RE, O'Donovan SM, Drummond JB, Benesh FS, Simmons M, Roberts $\mathrm{R}$, et al. Cell-specific abnormalities of glutamate transporters in schizophrenia: sick astrocytes and compensating relay neurons? Mol Psychiatry. 2016;21:823-30.

25. O'Donovan SM, Hasselfeld K, Bauer D, Simmons M, Roussos P, Haroutunian V, et al. Glutamate transporter splice variant expression in an enriched pyramidal cell population in schizophrenia. Transl Psychiatry. 2015;5:e579.

26. Sodhi MS, Simmons M, McCullumsmith R, Haroutunian V, Meador-Woodruff JH. Glutamatergic gene expression is specifically reduced in thalamocortical projecting relay neurons in schizophrenia. Biol Psychiatry. 2011;70:646-54.

27. Drummond JB, Tucholski J, Haroutunian V, Meador-Woodruff JH. Transmembrane AMPA receptor regulatory protein (TARP) dysregulation in anterior cingulate cortex in schizophrenia. Schizophr Res. 2013;147:32-8.

28. Kashihara K, Sato M, Fujiwara Y, Ogawa T, Fukuda K, Otsuki S. Effects of intermittent and continuous haloperidol administration on the dopaminergic system in the rat brain. Yakubutsu Seishin Kodo=Japanese J Psychopharmacol. 1986;6:275-80.

29. Zimmermann $\mathrm{H}$, Zebisch $\mathrm{M}$, Strater N. Cellular function and molecular structure of ecto-nucleotidases. Purinergic Signal. 2012;8:437-502.

30. Yegutkin GG. Nucleotide- and nucleoside-converting ectoenzymes: Important modulators of purinergic signalling cascade. Biochim Biophys Acta. 2008; 1783:673-94.

31. Corriden R, Insel PA. Basal release of ATP: an autocrine-paracrine mechanism for cell regulation. Sci Signal. 2010;3:re1.

32. Lu D, Insel PA. Hydrolysis of extracellular ATP by ectonucleoside triphosphate diphosphohydrolase (ENTPD) establishes the set point for fibrotic activity of cardiac fibroblasts. J Biol Chem. 2013;288:19040-9.

33. Dunwiddie TV, Masino SA. The role and regulation of adenosine in the central nervous system. Annu Rev Neurosci. 2001;24:31-55.

34. Choi DS, Cascini MG, Mailliard W, Young H, Paredes P, McMahon T, et al. The type 1 equilibrative nucleoside transporter regulates ethanol intoxication and preference. Nat Neurosci. 2004;7:855-61.

35. Shan D, Haroutunian V, Meador-Woodruff JH, McCullumsmith RE. Expression of equilibrative nucleoside transporter type 1 protein in elderly patients with schizophrenia. Neuroreport. 2012a;23:224-7.

36. Wall MJ, Dale N. Neuronal transporter and astrocytic ATP exocytosis underlie activity-dependent adenosine release in the hippocampus. J Physiol. 2013;591:3853-71.

37. Cristalli G, Costanzi S, Lambertucci C, Lupidi G, Vittori S, Volpini R, et al. Adenosine deaminase: functional implications and different classes of inhibitors. Med Res Rev. 2001;21:105-28.

38. Brunstein MG, Silveira EM Jr., Chaves LS, Machado $H$, Schenkel O, Belmonte-deAbreu $\mathrm{P}$, et al. Increased serum adenosine deaminase activity in schizophrenic receiving antipsychotic treatment. Neurosci Lett. 2007;414:61-4.

39. Dutra GP, Ottoni GL, Lara DR, Bogo MR. Lower frequency of the low activity adenosine deaminase allelic variant $(A D A 1 * 2)$ in schizophrenic patients. Rev Bras De Psiquiatr. 2010;32:275-8.

40. Shan D, Yates S, Roberts RC, McCullumsmith RE. Update on the neurobiology of schizophrenia: a role for extracellular microdomains. Minerva Psichiatr. 2012b;53:233-49.

41. Fredholm BB, Chen JF, Cunha RA, Svenningsson P, Vaugeois JM. Adenosine and brain function. Int Rev Neurobiol. 2005;63:191-270.

42. Gimenez-Llort L, Fernandez-Teruel A, Escorihuela RM, Fredholm BB, Tobena A, Pekny $M$, et al. Mice lacking the adenosine $A 1$ receptor are anxious and aggressive, but are normal learners with reduced muscle strength and survival rate. Eur J Neurosci. 2002;16:547-50.

43. McCullumsmith $\mathrm{RE}$, Hammond JH, Shan D, Meador-Woodruff JH. Postmortem brain: an underutilized substrate for studying severe mental illness. Neuropsychopharmacol: Off Publ Am Coll Neuropsychopharmacol. 2015;40:1307.

44. Zhang Y, Chen K, Sloan SA, Bennett ML, Scholze AR, O'Keeffe S, et al. An RNAsequencing transcriptome and splicing database of glia, neurons, and vascular cells of the cerebral cortex. J Neurosci: Off J Soc Neurosci. 2014;34:11929-47.

45. Seibt KJ, Oliveira Rda L, Bogo MR, Senger MR, Bonan CD. Investigation into effects of antipsychotics on ectonucleotidase and adenosine deaminase in zebrafish brain. Fish Physiol Biochem. 2015;41:1383-92.

46. Seibt KJ, Oliveira Rda L, Rico EP, Dias RD, Bogo MR, Bonan CD. Antipsychotic drugs inhibit nucleotide hydrolysis in zebrafish (Danio rerio) brain membranes. Toxicol Vitr: Int J Publ Assoc BIBRA. 2009;23:78-82. 